\title{
MORTALIDADE MATERNA NO ESTADO DO AMAZONAS: ESTUDO EPIDEMIOLÓGICO
}

\author{
MATERNAL MORTALITY IN THE STATE OF \\ AMAZONAS: AN EPIDEMIOLOGICAL STUDY
}

\section{MORTALIDAD MATERNA EN EL ESTADO DE AMAZONAS: ESTUDIO EPIDEMIOLÓGICO}

\author{
Lidiane Tavares Medeiros ${ }^{1}$ \\ António Manuel Sousa ${ }^{2}$ \\ Leudson Oliveira Arinana ${ }^{1}$ \\ Antônio Sávio Inácio ${ }^{1}$ \\ Maria do Livramento Coelho Prata ${ }^{3}$ \\ Milaine Nunes Gomes Vasconcelos ${ }^{3}$
}

Como citar este artigo: Medeiros LT, Sousa AM, Arinana LO, Inácio AS, Prata MLC, Vasconcelos MNG. Mortalidade materna no estado do Amazonas: estudo epidemiológico. Rev baiana enferm. 2018;(32):e26623.

Objetivo: analisar o perfil epidemiológico da mortalidade materna no estado do Amazonas, Brasil. Método: estudo epidemiológico, descritivo e ecológico, baseado em dados secundários do Sistema de Informação Sobre Mortalidade e Sistema de Informação Sobre Nascidos Vivos do Ministério da Saúde, gerados pelo Departamento de Análise e Tabulação de Dados do Sistema Único de Saúde. Utilizou-se os dados dos óbitos maternos e suas causas, além dos dados dos nascidos vivos do Amazonas, Brasil, do período de 2006-2015, sendo estes coletados em 2017. Resultados: ocorreram 564 óbitos maternos, sendo 329 na capital e 235 no interior, resultando um coeficiente de mortalidade materna de 73,45 óbitos/100.000 nascidos vivos. As principais causas de óbitos maternos foram: infecção puerperal, eclâmpsia e hemorragia pós-parto. Conclusão: as mulheres solteiras, entre 20-29 anos, pardas e baixa escolaridade apresentaram maior prevalência de óbito materno. Entre os óbitos, a infecção puerperal foi a mais evidenciada entre as causas obstétricas diretas.

Descritores: Mortalidade Materna. Perfil de Saúde. Infecção Puerperal. Eclâmpsia. Hemorragia Pós-parto.

Objective: to analyze the epidemiological profile of maternal mortality in the state of Amazonas, Brazil. Method: a descriptive and ecological epidemiological study was carried out based on secondary data from the Mortality Information System Database and Live Births Information System of the Brazilian Ministry of Health, generated by the Department of Data Analysis and Tabulation of the Brazilian Unified Health System. Data collection was carried out in 2017, using data from maternal deaths and their causes, in addition to data from live births of the state of Amazonas, Brazil, from 2006 to 2015. Results: the total number of maternal deaths was 564, being 329 in the capital city and 235 in the countryside, resulting in a maternal mortality ratio of 73.45 deaths per 100,000 live births. The main causes of maternal deaths were, as follows: puerperal infection, eclampsia, and postpartum hemorrhage. Conclusion: single women aged between 20 and 29 years, brown, and with a low education level presented a higher prevalence of maternal death. Puerperal infection stood out among the direct obstetric causes.

\footnotetext{
Estudante de Enfermagem, Universidade do Estado do Amazonas. Manaus, Amazonas, Brasil. Itm.enf@uea.edu.br

Biólogo. Doutor em Saúde Coletiva. Docente da Universidade do Estado do Amazonas. Manaus, Amazonas, Brasil. amzsouza@uea.edu.br

Enfermeira Obstétrica. Docente da Universidade do Estado do Amazonas. Manaus, Amazonas, Brasil. mngomes@uea.edu.br
} 
Objetivo: analizar el perfil epidemiológico de la mortalidad materna en el estado de Amazonas, Brasil. Método: estudio epidemiológico, descriptivo y ecológico, basado en datos secundarios del Sistema de Información sobre Mortalidad y sistema de Información sobre Nacidos Vivos del Ministerio de Salud, generados por el Departamento de Análisis y Tabulación de Datos del Sistema Único de Salud. Se utilizaron datos de óbitos maternos y sus causas, datos de nacidos vivos en Amazonas, Brasil de 2006-2015, recolectados en 2017. Resultados: hubo 564 óbitos maternos; 329 en la capital y 235 en el interior, determinando un coeficiente de 73,45 óbitos maternos/100.000 nacidos vivos. Las principales causas de óbito fueron: infección puerperal, eclampsia y hemorragia posparto. Conclusión: las mujeres solteras, de entre 20-29 años, mestizas y de baja escolarización expresaron mayor prevalencia de óbito materno. La infección puerperal fue la causa obstétrica directa más evidenciada entre los óbitos.

Descriptores: Mortalidad Materna. Perfil de Salud. Infección Puerperal. Eclampsia. Hemorragia Posparto.

\section{Introdução}

A Organização Mundial de Saúde (OMS) define mortalidade materna (MM) como morte da mulher ocasionada no período da gestação ou até 42 dias após o término desta, não interessando a duração ou localização da gravidez. Considera, ainda, qualquer causa associada ou agravada pela gestação ou por condutas tomadas em decorrência desse estado, excluindo apenas as causas acidentais ou incidentais, representadas pelas mortes violentas consumadas durante o período puerperal ${ }^{(1)}$.

Os óbitos maternos podem ser categorizados em causas obstétricas diretas, indiretas e não especificadas. A mortalidade materna direta é a relacionada com complicações exclusivamente ligadas ao ciclo gravídico-puerperal, podendo estar associada à má qualidade da assistência prestada à saúde da mulher na gestação, parto ou puerpério, comumente encontrada em países em desenvolvimento ${ }^{(2)}$. A mortalidade materna por causas obstétricas indiretas decorre de condições pré-existentes, isto é, patologias anteriores à gestação, que se exacerbam com a gravidez. Em ambos os tipos de classificação de mortalidade materna, é evidente que a má assistência à gestante acarreta um desfecho desfavorável. Essa morte poderia ser evitada mediante o desenvolvimento de ações de qualidade, tratamento precoce e eficaz ${ }^{(3)}$.

As causas de mortalidade materna direta estão codificadas pelo CID-10 como: 000.0 a 008.9, 011 a 023.9, 024.4, 026.0 a 092.7, D39.2, E23.0, F53 e M83.0. A mortalidade obstétrica indireta é codificada como: 010.0 a 010.9; 024.0 a 024.3; 024.9,
025, 098.0 a 099.8, A34, B20 a B24 (estes últimos após criteriosa investigação). Por último, a mortalidade obstétrica não especificada corresponde ao código $0.95^{(4)}$.

Para mensurar a mortalidade materna, sua extensão e predisposição no espaço e no tempo, é utilizado o Coeficiente de Mortalidade (CMM) como indicador de saúde. Esse indicador é avaliado pelo número de óbitos maternos a cada 100 mil nascidos vivos (NV), sendo aceitável pela OMS o limite de 20 mortes a cada 100 mil nascidos vivos ${ }^{(5)}$.

O CMM pode ser classificado como baixo, quando se tem até 20 óbitos por 100 mil NV; médio, quando o número de óbitos fica entre 20 e 49; alto, quando se tem 50 a 149 óbitos; e muito alto, quando o número é maior que 150 por 100 mil NV $^{(6)}$. Ressalta-se que o resultado desse cálculo não é totalmente fidedigno, pois há uma alta taxa de sub-registro de óbitos, bem como das causas da morte, impossibilitando o conhecimento da real magnitude desse indicador $^{(7)}$.

No Brasil, a mortalidade materna apresentou queda de 120 óbitos por 100 mil NV no ano de 1990, para 69 óbitos por 100 mil NV em 2013, exibindo queda de 43\% no CMM. Apesar da mudança, o Brasil ainda não atingiu o 50 objetivo do milênio, em que o CMM ficaria na margem de 35 mortes por 100 mil NV, nível aproximadamente duas vezes superior ao objetivo instituído ${ }^{(8)}$.

No Amazonas, Brasil, estudo realizado para avaliar a mortalidade no período de 2001 a 2010, mostrou a ocorrência de 6.932 mortes de 
mulheres em idade fértil. Desse total, 241 óbitos foram classificados como morte materna obstétrica, caracterizando um CMM de 63,2 para cada 100 mil NV. As mortes maternas obstétricas diretas (MMOD) refletiram $162(67,2 \%)$ casos, ao mesmo tempo em que as mortes maternas obstétricas indiretas (MMOI) registraram 62 (25,7\%) $\operatorname{casos}^{(9)}$.

O Amazonas possui 62 municípios cujo acesso, em sua grande maioria, só é possível pela via fluvial. As distâncias apresentam grande relevância, principalmente quando comparado Manaus (capital) com o interior. Como exemplo, a distância pela via fluvial entre Manaus e Lábrea, município do interior, é de $7.495 \mathrm{~km}$, quase a mesma entre o Rio de Janeiro, Brasil, até Lisboa, Portugal, que totaliza $7.711 \mathrm{~km}^{(10)}$. Devido às condições geográficas, dificuldade de acesso aos serviços de saúde e/ou notificações, principalmente da população do interior, as informações sobre a mortalidade materna podem estar subnotificadas, o que pode implicar no não conhecimento do número real dos óbitos ocorridos.

Diante do exposto, o estudo epidemiológico proposto poderá proporcionar o enriquecimento da comunidade acadêmica, com o aumento da produção científica na temática estudada, uma vez que há escassez de produções. Também será importante para despertar a necessidade de uma análise mais crítica sobre a assistência ofertada às mulheres, que poderia estar voltada para evitar ou reduzir as taxas de mortalidade materna. Para realizar a análise proposta, será identificado o coeficiente de mortalidade materna das mesorregiões do estado, descrito o perfil sociodemográfico das mulheres que sofreram o óbito materno, além de identificar as principais causas de óbitos no estado.

O estudo tem como objetivo analisar o perfil epidemiológico da mortalidade materna no estado do Amazonas, Brasil.

\section{Método}

Foi realizado um estudo epidemiológico, descritivo e ecológico, baseado em dados secundários do Sistema de Informação sobre Mortalidade
(SIM) e Sistema de Informação sobre Nascidos Vivos (SINASC) do Ministério da Saúde, gerados pelo Departamento de Análise e Tabulação de Dados do Sistema Único de Saúde (DATASUS) ${ }^{(11)}$.

A coleta ocorreu no ano de 2017, utilizando-se dados de todos os óbitos maternos do estado do Amazonas, registrados no período de 2006-2015, possibilitando a análise do comportamento da mortalidade materna em uma série histórica de 10 anos. Para caracterizar o perfil sociodemográfico, utilizou-se as variáveis: faixa etária, nível de escolaridade, raça/cor, estado civil e local de ocorrência. Referente à causa óbito, a variável de desfecho do estudo, utilizou-se a $10^{\mathrm{a}}$ revisão da classificação internacional de doenças (CID-10), com inclusão do Capítulo XV (Gravidez, Parto e Puerpério) e das categorias específicas relacionadas às causas obstétricas diretas, indiretas e não especificadas (B20-B24 e O00-O99). Na construção da série histórica de óbitos maternos (Manaus/Interior), e do coeficiente de mortalidade materna de cada mesorregião, foram utilizados como variáveis os valores absolutos do número de óbitos maternos, número de nascidos vivos e as mesorregiões do Amazonas (Norte, Centro, Sul e Sudoeste).

Para organização, os dados foram tabulados em planilha no Excel, posteriormente descritos em tabelas, gráficos e medidas numéricas como a média. Nas tabelas e gráficos, os valores foram apresentados em frequências absolutas e/ou relativas e por nível descritivo. Para cálculo de coeficiente de mortalidade materna foi utilizado o número de óbitos maternos, dividido pelo total de nascidos vivos de mães residentes no mesmo local e período x $10^{5}$. Para classificação em baixa, média, alta e muito alta, foram utilizados os parâmetros de CMM da OMS.

Em conformidade com a Resolução n. 466/2012, do Conselho Nacional de Saúde, não houve necessidade de submeter o projeto ao Comitê de Ética em Pesquisa (CEP), por se tratar de dados secundários, de domínio público, em que a identidade do sujeito da pesquisa não é determinada.

De acordo com o Instituto Brasileiro de Geografia e Estatística (IBGE), o Amazonas possuía 
uma população de 3.483 .985 habitantes em 2010 e estava dividido em quatro Mesorregiões e 62 municípios: Mesorregião Centro Amazonense, a qual compreende 30 municípios, a maior de toda as regiões; em seguida Mesorregião Sudoeste, com 16 municípios; Mesorregião Sul, com 10 municípios; e Mesorregião Norte, com 6 municípios $^{(12)}$.

\section{Resultados}

No período de 2006 a 2015, foram registrados 564 óbitos por mortalidade materna no Amazonas. Destes, 329 (58,33\%) ocorreram em Manaus, capital do estado, e 235 (41,67\%), nos demais municípios do interior, representados pela série histórica no Gráfico 1.

Gráfico 1 - Número de óbitos maternos ocorridos em Manaus e demais municípios. Amazonas, Brasil - 2006-2015 (N=564)

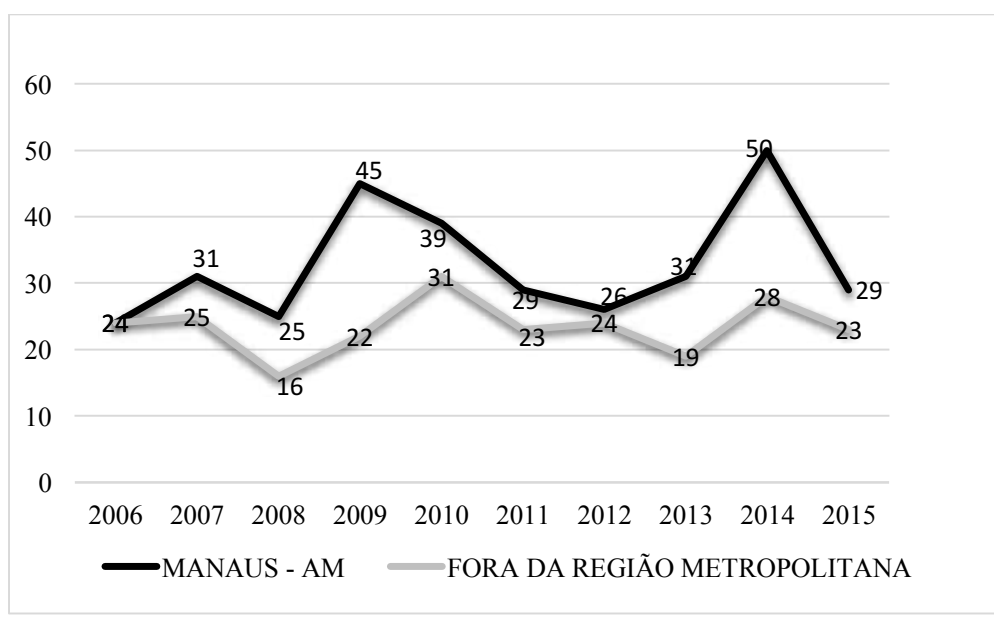

Fonte: Elaboração própria.

No período de 2006 a 2015, no estado do Amazonas, foram registrados 767.919 nascidos vivos. O coeficiente de mortalidade materna nesse mesmo período foi de 73,45 óbitos/100.000
NV, classificado como alto pela OMS. O número de óbitos, de NV e o CMM estão informados na Tabela 1.

Tabela 1 - Distribuição do número de óbitos maternos, número de nascidos vivos e Coeficiente de Mortalidade Materna. Amazonas, Brasil - 2006-2015 ( $\mathrm{N}=564$ )

\begin{tabular}{l|c|c|c|c}
\hline Ano & $\mathbf{n}$ & $\mathbf{\%}$ & Nascidos vivos & $\begin{array}{c}\text { Coeficiente de } \\
\text { Mortalidade por 10 }\end{array}$ \\
\hline 2006 & 48 & 8,51 & 75.584 & 63,51 \\
2007 & 56 & 9,93 & 73.469 & 76,22 \\
2008 & 41 & 7,30 & 75.030 & 54,64 \\
2009 & 67 & 11,87 & 75.729 & 88,47 \\
2010 & 70 & 12,41 & 74.188 & 94,35 \\
2011 & 52 & 9,22 & 76.202 & 68,24 \\
2012 & 50 & 8,86 & 77.434 & 64,57 \\
2013 & 50 & 8,86 & 79.041 & 63,26 \\
2014 & 78 & 13,82 & 81.145 & 96,12 \\
2015 & 52 & 9,22 & 80.097 & 64,92 \\
Total & 564 & 100,00 & 767.919 & 73,45 \\
\hline
\end{tabular}

Fonte: Elaboração própria. 
A média do CMM de cada Mesorregião do estado, no período de 10 anos, permaneceu alta. A Mesorregião com a maior média de CMM foi a Norte (112,77 óbitos/100.000 NV), seguida das Mesorregiões Sudoeste (90,94 óbitos/100.000 NV), Sul (82,96 óbitos/100.000 NV) e Centro (68,41 óbitos/100.000 NV).

No Gráfico 2, está apresentada a série histórica dos CMM por cada Mesorregião do Amazonas. O maior CMM ocorreu nas Mesorregiões Centro, Norte, Sudoeste e Sul nos respectivos anos de 2014 (90,71 óbitos/100.000 NV), 2013 (191,13 óbitos/100.000 NV), 2010 (199,40 óbitos/100.000 NV) e 2014 (143,22 óbitos/100.000 NV). O menor
CMM encontrado foi no ano de 2008 nas Mesorregiões Norte (0 óbitos/100.000 NV), Sudoeste $(45,23$ óbitos/100.000 NV) e Centro (55,04 óbitos/100.000 NV); e no ano de 2013 na Mesorregião Sul (0 óbitos/100.000 NV). Mesmo com esses menores valores encontrados, quando comparados na série histórica, as Mesorregiões Sudoeste e Centro permanecem com CMM classificados como médio e alto, respectivamente. Os dados revelam ainda que, mesmo que a maioria das Mesorregiões apresente CMM alto, a Mesorregião Norte destaca-se, apresentando os maiores coeficientes no período estudado.

Gráfico 2 - Coeficiente da Mortalidade Materna das Mesorregiões. Amazonas, Brasil - 2006-2015

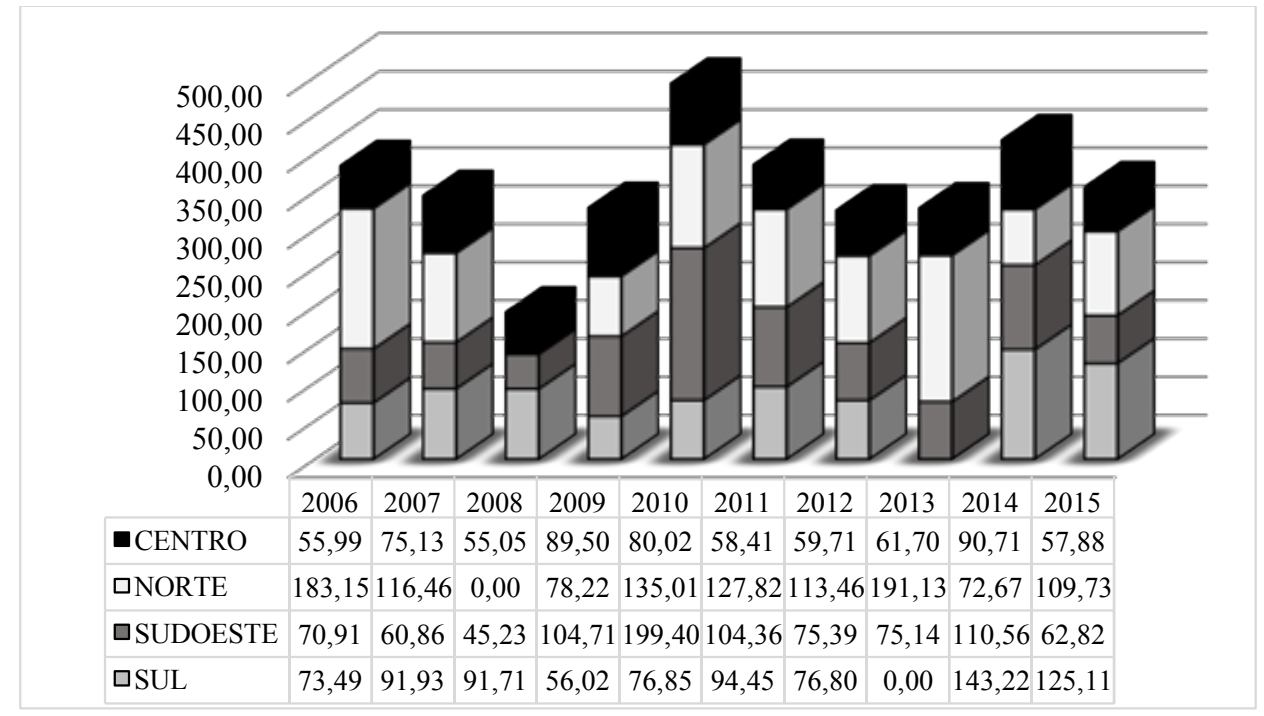

Fonte: Elaboração própria.

Na Tabela 2, está descrito o perfil sociodemográfico da mortalidade materna. A faixa etária prevalente para os óbitos maternos, tanto na capital ( $n=138 / 41,95 \%)$ como no interior $(n=93 / 39,57 \%)$, foi de 20 a 29 anos. O tempo de escolaridade de 4 a 7 anos e de 8 a 11 anos, foi encontrado no mesmo quantitativo para a capital: 107 (32,52\%) óbitos. Já no interior, para o mesmo tempo de 4 a 7 anos de estudo, o número de óbitos foi de
65 (27,66\%). O estado civil em maior destaque, tanto para a capital $208(63,22 \%)$ como para o interior 130 (55,32\%), foi o de solteira. A cor/ raça com maior taxa, na capital $236(71,73 \%)$ e interior 146 (62,13\%), foi a parda. O local de maior ocorrência dos óbitos maternos, tanto para capital 308 (93,62\%) como para interior 194 $(82,55 \%)$, foi o ambiente hospitalar. 
Tabela 2 - Caracterização dos óbitos maternos ocorridos na capital e no interior. Amazonas, Brasil 2006-2015 (N=564)

\begin{tabular}{|c|c|c|c|c|}
\hline \multirow{2}{*}{ Variáveis } & \multicolumn{2}{|c|}{ Capital } & \multicolumn{2}{|c|}{ Interior } \\
\hline & $\mathbf{N}=329$ & $\%$ & $\mathbf{N}=\mathbf{2 3 5}$ & $\%$ \\
\hline \multicolumn{5}{|l|}{ Faixa Etária } \\
\hline 10 a 14 anos & 5 & 1,52 & 4 & 1,70 \\
\hline 15 a 19 anos & 56 & 17,02 & 52 & 22,13 \\
\hline 20 a 29 anos & 138 & 41,95 & 93 & 39,57 \\
\hline 30 a 39 anos & 113 & 34,35 & 65 & 27,66 \\
\hline 40 a 49 anos & 17 & 5,17 & 21 & 8,94 \\
\hline \multicolumn{5}{|l|}{ Escolaridade } \\
\hline Nenhuma & 9 & 2,74 & 36 & 15,32 \\
\hline 1 a 3 anos & 31 & 9,42 & 34 & 14,47 \\
\hline 4 a 7 anos & 107 & 32,52 & 65 & 27,66 \\
\hline 8 a 11 anos & 107 & 32,52 & 53 & 22,55 \\
\hline 12 ou mais & 35 & 10,64 & 17 & 7,23 \\
\hline Formação Ignorada & 40 & 12,16 & 30 & 12,77 \\
\hline \multicolumn{5}{|l|}{ Estado Civil } \\
\hline Solteira & 208 & 63,22 & 130 & 55,32 \\
\hline Casadas & 69 & 20,99 & 45 & 19,15 \\
\hline Viúva & 1 & 0,30 & 1 & 0,43 \\
\hline Divorciada & 1 & 0,30 & - & - \\
\hline Outros & 16 & 4,86 & 42 & 17,87 \\
\hline Ignorado & 34 & 10,33 & 17 & 7,23 \\
\hline \multicolumn{5}{|l|}{ Raça } \\
\hline Branca & 64 & 19,45 & 22 & 9,36 \\
\hline Preta & 5 & 1,52 & 4 & 1,70 \\
\hline Amarela & 1 & 0,30 & - & - \\
\hline Parda & 236 & 71,73 & 146 & 62,13 \\
\hline Indígena & 5 & 1,52 & 60 & 25,53 \\
\hline Ignorado & 18 & 5,48 & 3 & 1,28 \\
\hline \multicolumn{5}{|l|}{ Local de Ocorrência } \\
\hline Hospital & 308 & 93,62 & 194 & 82,55 \\
\hline Domicílio & 16 & 4,86 & 33 & 14,04 \\
\hline Via Pública & 3 & 0,91 & - & - \\
\hline Outros & 2 & 0,61 & 8 & 3,40 \\
\hline
\end{tabular}

Fonte: Elaboração própria.

Nota: Sinal convencional utilizado:

- Dado numérico igual a zero não resultante de arredondamento.

Os óbitos por causas obstétricas diretas (379), indiretas (150) e não definidas (35) representaram, respectivamente, 67,19\%, 26,59\% e 6,20\% do total de óbitos maternos $(564$ - 100\%) registrados no estado.
Entre os óbitos maternos, as causas obstétricas diretas (379 - 100\%), indiretas (150 - 100\%) e não definidas (35 - 100\%) mais predominantes neste estudo foram, respectivamente: Infecção puerperal (86 - 22,69\%); outras Doenças Maternas 
Classificadas em outra Parte (COP) complicações gravidez/parto/puerpério (104 - 69,33\%); e Morte obstétrica de causas não especificadas (NE) (35 - 100\%), descritas na Tabela 3.

Tabela 3 - Principais causas de óbitos maternos, por categoria CID-10. Amazonas, Brasil - 2006-2015 $(\mathrm{N}=564)$

\begin{tabular}{|c|c|c|}
\hline CID-10/Principais Causa de Óbitos Maternos & $\mathbf{n}$ & $\%$ \\
\hline \multicolumn{3}{|l|}{ Obstétricas Diretas $(\mathrm{N}=379)$} \\
\hline O85 - Infecção Puerperal & 86 & 22,69 \\
\hline O15 - Eclampsia & 51 & 13,46 \\
\hline O72 - Hemorragia Pós-Parto & 29 & 7,65 \\
\hline O14 - Hipertensão Gestacional com Proteinúria Significativa & 21 & 5,54 \\
\hline O62 - Anormalidade da Contração Uterina & 19 & 5,01 \\
\hline O45 - Descolamento Prematuro de Placenta & 15 & 3,96 \\
\hline $\begin{array}{l}\text { O75 - Outras Complicações do Trabalho de Parto e Parto Não } \\
\text { Classificadas em Outra Parte }\end{array}$ & 13 & 3,43 \\
\hline O88 - Embolia de Origem Obstétrica & 13 & 3,43 \\
\hline O06 - Aborto Não Especificado & 12 & 3,17 \\
\hline Outros & 150 & 39,57 \\
\hline \multicolumn{3}{|l|}{ Obstétricas Indiretas $(\mathbf{N}=150)$} \\
\hline $\begin{array}{l}\text { O99 - Outras Doenças Maternas Classificados em Outra Parte } \\
\text { Complicações Gravidez/Parto/Puerpério }\end{array}$ & 104 & 69,33 \\
\hline $\begin{array}{l}\text { O98 - Doenças Infecciosas e Parasitárias Materna Classificadas em Outra } \\
\text { Parte, Complicações Gravidez/Parto/Puerpério }\end{array}$ & 27 & 18,00 \\
\hline B24 - Doença por HIV Não Especificada & 8 & 5,33 \\
\hline B20 - Doença por HIV resultado doença infecciosa e parasitária & 6 & 4,00 \\
\hline O10 - Hipertensão pré-existente Complicações Gravidez/Parto/Puerpério & 3 & 2,00 \\
\hline O24 - Diabetes Mellitus na Gravidez & 1 & 0,67 \\
\hline O25 - Desnutrição na Gravidez & 1 & 0,67 \\
\hline \multicolumn{3}{|l|}{ Obstétricas Não Especificadas $(\mathbf{N}=35)$} \\
\hline O95 - Morte obstétrica de causa Não Especificada & 35 & 100 \\
\hline
\end{tabular}

Fonte: Elaboração própria.

As categorias B20 (Doenças para HIV resultado doenças infecciosas e parasitárias) e B24 (Doença para HIV NE) não estão inseridas no capítulo XV (Gravidez, Parto e Puerpério), porém foram analisadas como causas obstétricas indiretas de óbitos de maternos.

Observando todos os óbitos maternos da série histórica estudada (2006 a 2015), a infecção puerperal destacou-se, apresentando maior taxa $(22,69 \%)$ entre as causas obstétricas diretas. Na distribuição anual desses óbitos, houve maior taxa nos anos de 2006 (20,00\%), 2007 (26,83\%), 2008 (38,46\%), 2009 (34,69\%), 2010 (38,30\%) e 2011 (29,03\%) para infecção puerperal; em 2012
$(24,14 \%), 2013(19,44 \%)$ e $2015(18,75 \%)$ para eclampsia; e em 2014 (14,58\%) para hemorragia pós-parto.

Concernente aos óbitos maternos por causas obstétricas indiretas, foram encontradas as maiores taxas no período de 2006 a 2012, representando 74 (72,5\%) dos óbitos para Outras Doenças Maternas COP Complicações Gravidez/ Parto/Puerpério (O-99), bem como 16 (69,57\%) dos óbitos no ano de 2014 e 11 (64,71\%) dos óbitos no ano de 2015. Em 2013, a maior taxa observada foi $4(50,00 \%)$ para Doenças Infecciosas e Parasitárias Maternas COP Complicações Gravidez/Parto/Puerpério (O-98). 


\section{Discussão}

O estudo verificou que, no estado do Amazonas, nos anos de 2006 a 2015, o CMM de 73,45 óbitos/100 mil NV foi considerado alto, conforme classificação da OMS. Os CMM das Mesorregiões (Centro, Sudoeste, Norte e Sul), no período citado, não passaram por grandes variações, mantendo-se entre muito alto e alto. Contudo, predominou a classificação alta, isto é, a CMM entre 50 e 149 óbitos por 100 mil NV $^{(6)}$. Corrobora esses resultados, estudo realizado no Pará, no período de 2006 a 2010, ao mostrar que o CMM 51,9 óbitos/100 mil NV também foi alto, não havendo redução significativa no período ${ }^{(5)}$.

A alta taxa de mortalidade pode ser evitada pela oferta e garantia de acesso aos serviços de saúde, atendimento por profissionais qualificados, infraestrutura de trabalho e fortalecimento das políticas públicas de saúde. O desconhecimento em profundidade dessa realidade e também do impacto da mortalidade materna, principalmente devido ao sub-registro e/ou subnotificação de óbitos, dificultam nas ações para a redução da mortalidade ${ }^{(13)}$. Este fato é ainda mais preocupante quando se refere ao Amazonas, por sua complexidade geográfica, ao considerar sua extensão territorial e o deslocamento quase sempre por via fluvial. Isso reflete-se nas condições de acesso aos serviços de saúde, contribuindo como fator de vulnerabilidade para determinadas populações, além de poder resultar em desfechos desfavoráveis, como os óbitos maternos. Esta realidade pode ser observada nos CMM de cada Mesorregião do estado descrita neste estudo.

Na descrição do perfil sociodemográfico dos óbitos maternos deste estudo, observou-se que tanto na capital como no interior do estado as mulheres eram jovens, com baixa escolaridade, solteiras e pardas. Estudos em alguns estados do Brasil apresentaram perfis diferentes, mas, em relação à faixa etária, os dados são similares, demonstrando que a mortalidade materna ocorre em mulheres jovens (20 a 29 anos) ${ }^{(14-16)}$. As características sociodemográficas encontradas em estudo realizado em Fortaleza (CE) foram de óbitos que ocorreram com maior proporção em mulheres com baixa escolaridade - primeiro grau incompleto - $(24-42,90 \%)$, em mulheres pardas $(35-62,50 \%)$, e que viviam com seu companheiro $(30-53,60 \%)^{(16)}$. No Rio Grande do Sul, foi observado que a maior faixa etária aumentava as possibilidades de morte materna ${ }^{(17)}$, o que contradiz os resultados do estudo ora apresentado.

Em relação à escolaridade, estudos atuais apresentaram resultados similares ao deste estudo, ao informar que mulheres com menos tempo de escolaridade, em especial aquelas que possuíam menos de sete anos, foram mais frequentes nos casos de óbitos maternos ${ }^{(18)}$. Estudo mostrou que, nos casos de maior nível de escolaridade, menor foi o CMM, sendo a baixa escolaridade considerada um fator de risco ${ }^{(3)}$.

De acordo com o IBGE, em 2015, a população brasileira autodeclarou-se em cinco raças/ cores: brancos $(45,2 \%)$, pardos $(41,1 \%)$, pretos $(8,9 \%)$, amarelos $(0,5 \%)$ e indígenas $(0,4 \%)^{(19)}$.

Neste estudo, a raça/cor predominante dos óbitos maternos foi a parda. O mesmo resultado foi encontrado em estudo realizado no Brasil em 2012, no qual mulheres de cor parda representavam $42,74 \%$ dos óbitos maternos ${ }^{(18)}$. Estudo realizado no estado do Mato Grosso revela que mulheres pretas e pardas estão mais sujeitas ao óbito materno, não em decorrência da cor/raça ser um fator de risco, mas pelo fato de as desigualdades sociais e os acessos aos serviços de saúde tornarem-se uma vulnerabilidade ${ }^{(14)}$. Ressalta-se que a classificação de raça/cor é complexa, dada a grande miscigenação existente no país.

Outra característica importante no perfil sociodemográfico foi o estado civil. Entre os óbitos maternos encontrados, a maior taxa foi em mulheres solteiras, característica comum encontrada em outros estudos sobre a temática ${ }^{(3)}$. Estudos mostraram maior frequência de mulheres solteiras, podendo estar relacionado à desestruturação familiar, quebra de vínculo entre mãe e pai do bebê, bem como à falta de apoio das famílias. No estado de Pernambuco, por exemplo, os dados sociodemográficos informam que $68,2 \%$ 
dos óbitos maternos foram de mulheres solteiras, e estas apresentavam maior probabilidade de óbito, supondo que abandono do parceiro ou falta do apoio social, do qual o companheiro está inserido, favoreça os números elevados de óbitos maternos ${ }^{(17,20)}$.

O local de maior ocorrência dos óbitos maternos, igualmente observado na capital e no interior, foi o ambiente hospitalar. Resultado semelhante foi observado no estudo realizado em São Paulo, onde 98,5\% dos óbitos maternos ocorreram em ambiente hospitalar, indicando que há a necessidade de se avaliar o motivo desses desfechos, seja por falha no atendimento, seja por ausência de diagnóstico precoce das doenças que surgiram ou foram agravadas pela gestação ${ }^{(21)}$.

A mortalidade materna continua sendo um problema de saúde pública em países subdesenvolvidos e em desenvolvimento, inclusive no Brasil. Elementos, como falhas no planejamento e estratégias políticas dos profissionais de saúde contribuíram para o Brasil não atingir a meta do milênio de redução do CMM em três quartos ${ }^{(13)}$. As características sociodemográficas têm sido apontadas como possíveis fatores de risco para tal agravo, levando à necessidade de repensar estratégias para diminuir o número de óbitos maternos $^{(16)}$.

O estudo mostrou que, nos anos de 2006 a 2015, as causas obstétricas diretas foram responsáveis por mais da metade dos óbitos maternos no estado do Amazonas. Estudos realizados em Ribeirão Preto (SP) e na Bahia confirmaram esse resultado, mostrando a predominância de altas taxas para óbitos por causas obstétricas $\operatorname{diretas}^{(15,22)}$

No Brasil, os óbitos maternos com maior incidência são os de causas obstétricas diretas, caracterizando um dado importante, por serem considerados evitáveis e passíveis de prevenção. As causas obstétricas indiretas apresentaram menores taxas, e são de monitoramento difícil em decorrência de doenças graves, muitas vezes inevitáveis, associadas à gestação ${ }^{(23)}$.

As causas obstétricas diretas mais frequentes neste estudo foram: Infecção Puerperal,
Eclâmpsia e Hemorragia Pós-Parto. Em Ribeirão Preto (SP) estudo mostrou, na distribuição de causas obstétricas diretas de óbitos maternos, que a hipertensão representou 44,8\% das mortes avaliadas. No estado do Pará, a primeira causa de morte materna foi a hipertensão, com 34,6\%, seguida da hemorragia, com 22,2\% dos $\operatorname{casos}^{(5,15)}$.

Nas causas obstétricas indiretas, foram identificadas: Outras Doenças Maternas COP Complicações Gravidez/Parto/Puerpério e Doenças Infecciosas e Parasitárias Materna COP Complicações Gravidez/Parto/Puerpério. Foi identificado, no Pará, a distribuição de causas indiretas: 20\% por hipertensão arterial pré-existente à gestação e 20\% por doenças do aparelho circulatório ${ }^{(5)}$. Em Ribeirão Preto (SP), as doenças do aparelho respiratório $(12,1 \%)$ destacaram-se como causas obstétricas indiretas ${ }^{(15)}$.

Entre os estudos analisados, a infecção (e não especificamente a puerperal) foi citada como resultado em apenas um estudo realizado em Aracaju (SE), sobre evolução temporal da mortalidade materna no período de 2000 a 2010. Encontrou-se que as causas de óbitos maternos mais prevalentes foram transtornos hipertensivos (20,7\%), hemorragias (17,2\%) e Infecções $(17,2 \%)^{(24)}$. Comparando-se com o presente estudo, fica evidente a divergência de resultados, pois, neste, a maior prevalência encontrada nos óbitos maternos por causa obstétrica direta foram para infecção puerperal, seguida da eclampsia e da hemorragia pós-parto.

A limitação do estudo decorreu da dificuldade de correlacionar os dados obtidos no Sistema de Informação de Mortalidade com a realidade local, considerando a dificuldade de acesso e atendimento aos serviços de saúde, principalmente nos municípios mais distantes e remotos do estado do Amazonas. Entretanto, os artigos encontrados foram satisfatórios para a análise dos resultados do estudo.

\section{Conclusão}

Os resultados desta pesquisa permitiram concluir que mulheres solteiras, de faixa etária entre 20-29 anos, pardas e com 4 a 7 anos de 
escolaridade apresentaram maior prevalência de óbito materno, tanto para a população da capital, como para a população do interior do estado. $\mathrm{O}$ CMM no estado do Amazonas, de acordo com a OMS, foi classificado como alto. No número de óbitos maternos encontrados na série histórica, houve destaque para causas obstétricas diretas, sendo a infecção puerperal a mais evidenciada no presente estudo. Os óbitos por causas obstétricas diretas podem ser evitados, mediante melhoria da assistência às mulheres, porém o cenário da desigualdade social e econômica ainda é presente em países em desenvolvimento, tornando-se ainda mais expressivo quando agregado à complexidade geográfica, como ocorre no Amazonas.

\section{Colaborações:}

1. concepção, projeto, análise e interpretação dos dados: Lidiane Tavares Medeiros e Milaine Nunes Gomes Vasconcelos;

2. redação do artigo e revisão crítica relevante do conteúdo intelectual: Lidiane Tavares Medeiros, Antônio Sávio Inácio e Milaine Nunes Gomes Vasconcelos;

3. aprovação final da versão a ser publicada: Lidiane Tavares Medeiros, António Manuel Sousa, Leudson Oliveira Arinana, Antônio Sávio Inácio, Maria do Livramento Coelho Prata e Milaine Nunes Gomes Vasconcelos.

\section{Referências}

1. Alves MMR, Alves SV, Antunes MBC, Santos DLP. Causas externas e mortalidade materna: proposta de classificação. Rev Saúde Pública [Internet]. 2013 [cited 2017 Aug 10];47(2):283-91. Available from: DOI:10.1590/S0034-8910.2013047003642

2. Tognini S. Tendência da mortalidade materna na região do Grande ABC Paulista de 1997 a 2011 [dissertação]. São Paulo (SP): Universidade de São Paulo; 2014.

3. Sousa DMN, Mendes IC, Oliveira ET, Chagas ACMA, Catunda HLO, Oriá MOB. Mortalidade materna por causas hipertensivas e hemorrágicas: análise epidemiológica de uma década. Rev Enferm UERJ [Internet]. 2014 [cited 2017 Aug
10];22(4):500-6. Available from: http://www.epublicacoes.uerj.br/index.php/enfermagemuerj/ article/view/15314/11598

4. Brasil. Ministério da Saúde. Secretaria de Políticas de Saúde. Área Técnica de Saúde da Mulher. Manual dos Comitês de Mortalidade Materna. 2a ed. Brasília; 2002.

5. Botelho NM, Silva IFMM, Tavares JR, Lima LO. Causas de morte materna no estado do Pará, Brasil. Rev Bras Ginecol Obstet [online]. 2014 [cited 2017 Aug 10];36(7):290-5. Available from: DOI:10.1590/ SO100-720320140004892

6. Brasil. Ministério da Saúde. Superintendência de Políticas de Saúde. Coordenadoria de Gestão da Informação em Saúde. Manual de indicadores e parâmetros da saúde [Internet]. Brasília; 2010 [cited 2017 Ago 22]. Available from: www.saude. mt.gov.br/arquivo/2901

7. Szwarcwald CL, Escalante JJC, Rabello Neto DL, Souza Junior PRB, Victora CG. Estimação da razão de mortalidade materna no Brasil, 20082011. Cad Saúde Pública [online]. 2014 [cited 2017 Aug 25];30(suppl1):S71-S83. Available from: DOI: http://dx.doi.org/10.1590/0102-311X00125313

8. Resende LV, Rodrigues RN, Fonseca MC. Mortes maternas em Belo Horizonte, Brasil: percepções sobre qualidade da assistência e evitabilidade. Rev Panam Salud Publica [Internet]. 2015 [cited 2017 Sep 4];37(4):218-24. Available from: https:// scielosp.org/pdf/rpsp/v37n4-5/v37n4-5a05.pdf

9. Ramos SCS. Análise de casos de mortes maternas em Manaus nos anos de 2001 a 2010 [dissertação]. [Internet]. São Paulo (SP): Universidade Estadual Paulista; 2013 [cited 2017 Sep 13]. Available from: https://repositorio.unesp.br/handle/11449/106369

10. Simas DCS, Lima JS. Desafios da inclusão digital no interior do Amazonas e a internet como ferramenta de redução das desigualdades sociais e regionais. Trabalho apresentado no $2^{\circ}$ Congresso Internacional de Direito e Contemporaneidade: mídias e direitos da sociedade em rede. Santa Maria (RS); 2013. Anais (online). Santa Maria: UFSM, CCSH; 2013 [cited 2017 Sep 13]. Available from: http://www.ufsm.br/congressodireito/anais

11. Brasil. Ministério da Saúde. Portal da Saúde. Departamento de Informática do Sistema Único de Saúde. DATASUS. Informações de Saúde (TABNET). Mortalidade materna [Internet]. Brasília; 2016 [cited 2017 Sep 15]. Available from: 
Lidiane Tavares Medeiros, António Manuel Sousa, Leudson Oliveira Arinana, Antônio Sávio Inácio, Maria do Livramento Coelho Prata, Milaine Nunes Gomes Vasconcelos

http://www2.datasus.gov.br/DATASUS/index. php?area $=02$

12. Instituto Brasileiro de Geografia e Estatística. Brasil/Amazonas. Panorama [Internet]. Rio de Janeiro; 2010 [cited 2017 Sep 15]. Available from: https://cidades.ibge.gov.br/brasil/am/panorama

13. Dias JMG, Oliveira APS, Cipolotti R, Monteiro BKSM, Pereira RO. Mortalidade materna. Rev Med Minas Gerais [Internet]. 2015 [cited 2017 Sep 15];25(2):173-9. Available from: DOI: $10.5935 / 2238-3182.20150034$

14. Teixeira NZF, Pereira WR, Barbosa DA, Vianna LAC. Mortalidade materna e interface com a raça em Mato Grosso. Rev Bras Saúde Matern Infant [Internet]. 2012 [cited 2017 Sep 28];12(1):27-35. Available from: DOI: http://dx.doi.org/10.1590/ S1519-38292012000100003

15. Fernandes BB, Nunes FBBF, Prudêncio PS, Mamede FV. Pesquisa epidemiológica dos óbitos maternos e o cumprimento do quinto objetivo de desenvolvimento do milênio. Rev Gaúcha Enferm [Internet]. 2015 [cited 2017 Oct 6];36(esp):192-9. Available from: DOI: http:// dx.doi.org/10.1590/1983-1447.2015.esp.56792

16. Áfio ACE, Araújo MAL, Rocha AFB, Andrade RFV, Melo SP. Óbitos maternos: necessidade de repensar estratégias de enfrentamento. Rev Rene [Internet]. 2014 [cited 2017 Oct 6];15(4):631-8. Available from: DOI: $10.15253 / 2175-6783.2014000400010$

17. Carreno I, Bonilha ALL, Costa JSD. Perfil epidemiológico das mortes maternas ocorridas no Rio Grande do Sul, Brasil: 2004-2007. Rev Bras Epidemiol [Internet]. 2012 [cited 2017 Oct 18];15(2):396-406. Available from: DOI: http:// dx.doi.org/10.1590/S1415-790X2012000200017

18. Ferraza L, Bordignonb M. Mortalidade materna uma realidade que precisa melhorar. Rev baiana saúde pública [Internet]. 2012 [cited 2017 Oct
27];36(2):527-38. Available from: http://files.bvs. br/upload/S/0100-0233/2012/v36n2/a3253.pdf

19. Instituto Brasileiro de Geografia e Estatística. Cor ou raça (Brasil, 2015) [Internet]. Rio de Janeiro; 2015 [cited 2017 Oct 20]. Available from: https:// teen.ibge.gov.br/sobre-o-brasil/populacoa/corou-raca.html

20. Wanderley RMM, Vasconcelos Neto PJA, Pinheiro HDM. Perfil da mortalidade materna. Rev enferm UFPE on line. 2017 [cited 2017 Out 20];11(Supl. 4):1616-24. Available from: DOI: 10.5205/ reuol.10438-93070-1-

21. Vega CEP, Soares VMN, Nasr AMLF. Mortalidade materna tardia: comparação de dois comitês de mortalidade materna no Brasil. Cad Saúde Pública [online]. 2017 [cited 2017 Nov 3];33(3):e00197315. Available from: DOI: 10.1590/0102-311X00197315

22. Coelho VC, Andrade MS, Sena CD, Costa LEL, Bittencourt IS. Caracterização dos óbitos maternos em três regiões de saúde do centro-norte baiano. Cogitare Enferm [Internet]. 2016 [cited 2017 Nov 3];21(1):1-8. Available from: DOI: http://dx.doi. org/10.5380/ce.v21i1.42475

23. Castro BMC, Ramos SCS. Perfil de mortalidade materna em uma maternidade pública da cidade de Manaus-Am. Rev Saúde (Santa Maria) [Internet]. 2016 [cited 2017 Nov 5];42(1):103-12. Available from: https://periodicos.ufsm.br/revistasaude/article/ view/20953

24. Borges CLMS, Costa MCN, Mota ELA, Menezes GMS. Evolução temporal e diferenciais intra-urbanos da mortalidade materna em Aracaju, Sergipe, 20002010. Epidemiol Serv Saúde [Internet]. 2013 [cited 2017 Nov 5];22(2):307-16. Available from: DOI: 10.5123/S1679-49742013000200012

Recebido: 24 de maio de 2018

Aprovado: 1 de outubro de 2018

Publicado: 23 de dezembro de 2018

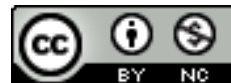

A Revista Baiana de Enfermagem utiliza a Licença Creative Commons - Atribuição-NãoComercial 4.0 Internacional. https://creativecommons.org/licenses/by-nc/4.0/ Este artigo é de acesso aberto distribuído sob os termos da Licença Creative Commons (CC BY-NC). Esta licença permite que outros remixem, adaptem e criem a partir do seu trabalho para fins não comerciais. Embora os novos trabalhos tenham de lhe atribuir o devido crédito e não possam ser usados para fins comerciais, os usuários não têm de licenciar esses trabalhos derivados sob os mesmos termos. 